\title{
Clinicopathologic features of remnant gastric cancer after curative distal gastrectomy according to previous reconstruction method: a retrospective cohort study
}

\author{
Yong-Eun Park and Sang-Woon Kim ${ }^{*}$ (1)
}

\begin{abstract}
Background: Survival rate of patients treated for gastric cancer has increased due to early detection and improvements of surgical technique and chemotherapy. Increase in survival rate has led to an increase in the risk for remnant gastric cancer (RGC). The purpose of this study was to investigate clinicopathologic features of RGC according to previous reconstruction method and factors affecting the interval from previous curative distal gastrectomy for gastric cancer to RGC occurrence.

Methods: Medical records of patients diagnosed with RGC at Yeungnam University Medical Center from January 2000 to December 2017 who had a history of distal gastrectomy with D2 LN dissection due to gastric cancer were reviewed retrospectively.

Results: Forty-eight patients were enrolled in this study. The mean interval of 48 RGC patients was 105.6 months (8.8 years). RGC after Billroth II reconstruction recurred more often at anastomosis site than RGC after Billroth I reconstruction $(p=0.001)$. The mean interval of RGC after Billroth I reconstruction was 67 months, shorter than 119 months of RGC after Billroth II reconstruction $(p=0.003)$. On the contrary, interval showed no difference according to stage of previous gastric cancer, remnant gastric cancer, or sex ( $p=0.810,0.145$, and 0.372 , respectively).

Conclusions: RGC after Billroth I reconstruction tends to arise earlier at non-anastomosis site than RGC after Billroth II. Therefore, we should examine non-anastomosis site carefully from the beginning of surveillance after gastric cancer surgery with Billroth I reconstruction for better outcome.
\end{abstract}

Keywords: Remnant gastric cancer, Reconstruction, Recurrence interval

\section{Introduction}

Gastric cancer is the fifth most common cancer and the third cause of death due to cancer in the world [1]. It is especially common in the Republic of Korea and Japan compared with its population. It is also the most common cancer in Korean males [2].

Remnant gastric cancer (RGC) is a carcinoma that develops in the remnant stomach after gastrectomy. RGC was first reported by Balfour without an exact nomenclature in 1922 as gastric cancer developing in patients

\footnotetext{
* Correspondence: swkim@medical.yu.ac.kr

Department of surgery, Yeungnam University Medical Center, Daegu, Korea
}

operated for gastric ulcer [3]. Since the report by Balfour, carcinoma arising from remnant stomach after gastrectomy due to gastric cancer was also included in RGC. Its nomenclature and definition was not unified for a long period. Researchers have used various terms such as stump gastric cancer, gastric remnant cancer, and carcinoma in remnant stomach with un-unified definition about the initial disease and duration from previous gastrectomy. Some researchers defined RGC as gastric cancer detected more than 5 years after gastric cancer surgery while other researchers defined it as gastric cancer detected more than 10 years after gastric

(c) The Author(s). 2019 Open Access This article is distributed under the terms of the Creative Commons Attribution 4.0 International License (http://creativecommons.org/licenses/by/4.0/), which permits unrestricted use, distribution, and 
cancer surgery $[4,5]$. Currently, RGC is defined as carcinoma arising from remnant stomach regardless of the initial disease or duration from previous surgery [6].

Recently, RGC after benign disease (RGC-B) has not increased as peptic ulcer gastrectomy has become rare due to development of PPI [7]. On the other hand, RGC after gastric cancer (RGC-M) has increased due to increased survival rate of gastric cancer patients because of early detection and improvement in surgical technique and chemotherapy [8]. Therefore, it is necessary to study clinicopathologic features of RGC after curative distal gastrectomy and establish reasonable follow-up examination plan for early detection of RGC which is essential for good prognosis.

This study was designed to examine clinicopathologic features of RGC-M based on previous reconstruction method and investigate clinicopathologic features affecting the interval from previous curative distal gastrectomy for gastric cancer to RGC occurrence.

\section{Methods}

Patients and study design

Among 4284 patients who underwent gastrectomy at Yeungnam University Medical Center (YUMC) from January 01, 2000 to December 31, 2017, medical records of 66 gastric cancer patients who had a history of gastrectomy were reviewed retrospectively.

Patients who fulfilled the following criteria were eligible in this study: (1) previous gastrectomy for gastric cancer, (2) regular follow-up studies before diagnosis with RGC, and (3) D2 LN dissection with Billroth I or Billroth II reconstruction method. According to the eligible criteria, data of 48 patients were analyzed retrospectively after excluding 18 patients who failed to meet these criteria (Fig. 1).

\section{Decision of reconstruction method in distal gastrectomy}

After D2 LN dissection was completed, great curvature on distal body of stomach was opened for visualization of the cancer lesion. The final decision on reconstruction method was done with reference to macroscopic findings and the location of the cancer to make tensionfree anastomosis. Anastomosis between the duodenum or jejunum and the posterior wall of the remnant body of the stomach (close to greater curvature) was then performed.

\section{Operation method for RGC}

Operation for 48 RGC patients was total gastrectomy with Roux-en-Y anastomosis. During RGC operation, residual peri-gastric LN dissection was done and LN 10 was removed in case of visible LN enlargement or RGC on greater curvature. If previous reconstruction method was Billroth II and infiltration of jejunum or LN

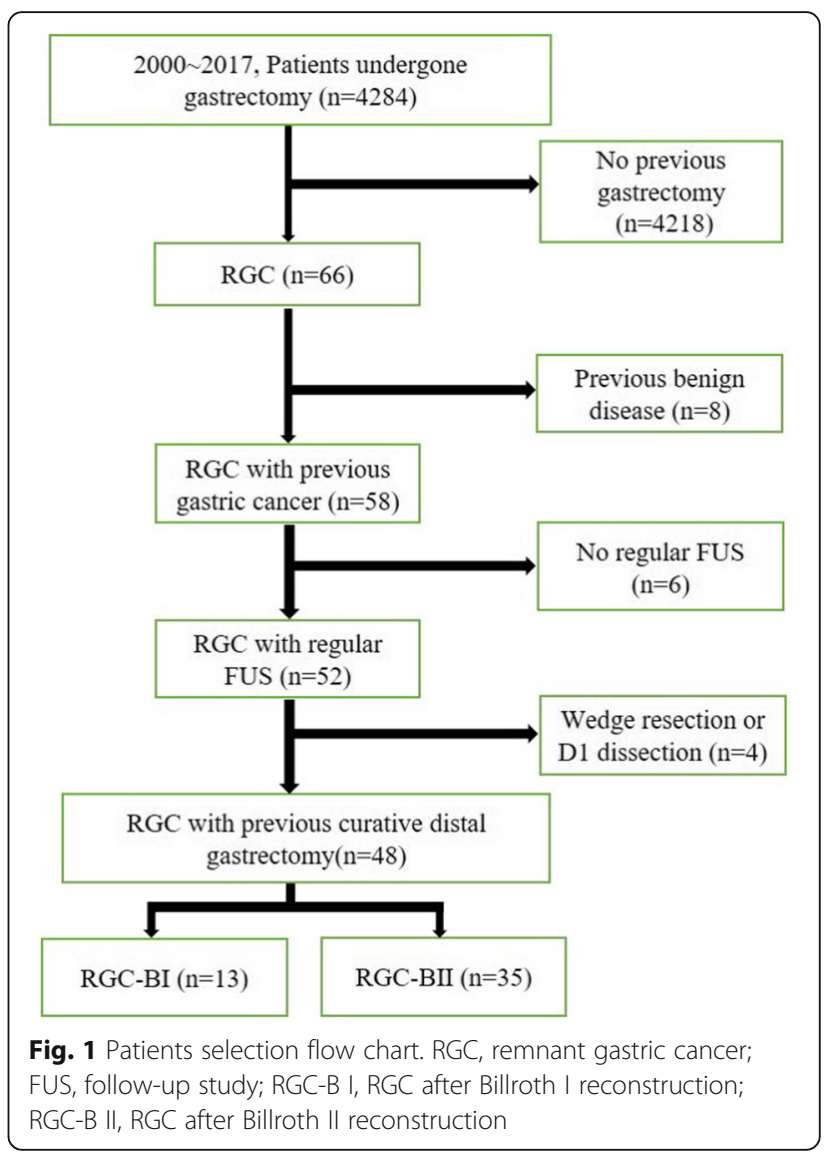

metastasis was suspected, jejuno-mesenteric LN dissection was performed, including the origin of each involved jejunal artery. Splenectomy was performed if patients met the following criteria: (1) uncontrolled bleeding of spleen, (2) direct invasion of spleen, and (3) invasion of gastro-splenic ligament.

\section{Follow-up study after gastrectomy}

For follow-up clinical studies, history taking, physical examination, serum tumor marker evaluation, simple chest $\mathrm{X}$ ray, esophagogastroduodenoscopy (EGD), and abdominal CT scan were carried out at intervals of 6 months for gastric carcinoma patients until the second year. Afterward, annual follow-up was performed until the 7th year for early gastric cancer and 10th year for advanced gastric cancer. If necessary, abdominal ultrasonic examination, chest CT scan, whole body bone scan, and PET scan were performed. After that, followup studies including EGD were carried out every two years. If patients agreed, annual follow-up studies continued.

\section{Variables}

Stages of RGC and previous gastric cancer were determined according to the 8th edition of the American 
Joint Commission on Cancer (AJCC). However, substage was not divided due to small sample size. Interval was defined as the time (month) from the previous gastrectomy to RGC surgery. Recurrence site was classified as anastomosis site (gastro-duodenostomy or gastrojejunostomy) and non-anastomosis site. Differentiation was determined by WHO classification and categorized into two groups. The differentiated group included papillary, well or moderately differentiated adenocarcinoma. The undifferentiated group included poorly differentiated adenocarcinoma, mucinous carcinoma, signet ring cell, or cohesive carcinoma. Pathologic report of RGC was reviewed and RGC was classified as intestinal, diffuse, or mixed type according to Lauren classification.

This study was conducted in accordance with the Declaration of Helsinki. It was approved by the Institutional Review Board of Yeungnam University Medical Center, Daegu, Republic of Korea (IRB No. 2018-09-030-001).

\section{Statistical analysis}

All statistical analyses were performed using SPSS version 22.0 (IBM Corporation, Armonk, NY, USA) with significance level set at $p<0.05$. Continuous variables are expressed as mean \pm standard deviation (range) and compared using Student's $t$ test and Cohen's $d$ with 95\% confidence interval. Categorical variables are expressed as frequency and compared using chi-square test or Fisher's exact test and odds ratio (OR) with 95\% confidence interval.

\section{Results}

The mean age of 48 RGC-M patients was 64.3 years (range, 39 to 80 years). There were $42(87.5 \%)$ males and $6(12.5 \%)$ females. Twenty-one (43.7\%) cases recurred at non-anastomosis site and $27(56.2 \%)$ cases recurred at anastomosis site. The mean length of the previous proximal resection margin $(\mathrm{PRM})$ was $5.9 \pm 3.8 \mathrm{~cm}$ and the mean interval of 48 RGC-M patients was $105.6 \pm 74.7$ months (Table 1).

Interval of RGC-M according to Lauren classification was 138.5 months for intestinal type, 76.2 months for diffuse type, and 103 months for mixed type, demonstrating statistically significant difference $(p=0.044$, Cohen's $d=$ - 0.805, - 0.474). Interval of RGC-M in anastomosis site was 132.6 months, which was longer than that (70.8 months) of RGC-M in non-anastomosis site ( $p=0.002$, Cohen's $d=0.953$ ). There was no significant difference in the interval by previous PRM or stage of previous gastric cancer or stage of RGC. There was no significant difference in the interval based on sex or differentiation of RGC either (Table 2).

The interval of RGC after Billroth I reconstruction (RGC-B I) was 67 months (5.5 years), which was shorter than that (119 months or 9.9 years) of RGC after Billroth
Table 1 Baseline characteristics of RGC patients after gastric cancer

\begin{tabular}{|c|c|}
\hline Variables & $(n=48)$ \\
\hline Age (years) & $64.3 \pm 8.9(39-80)$ \\
\hline \multicolumn{2}{|l|}{ Sex } \\
\hline Male & $42(87.5 \%)$ \\
\hline Female & $6(12.5 \%)$ \\
\hline \multicolumn{2}{|l|}{ Interval } \\
\hline$<5$ years & $15(31.3 \%)$ \\
\hline$\geq 5,<10$ years & $15(31.3 \%)$ \\
\hline$\geq 10$ years & $18(37.5 \%)$ \\
\hline Total (months) & $105.6 \pm 74.7(14-350)$ \\
\hline \multicolumn{2}{|c|}{ Reconstruction method } \\
\hline Billroth I & $13(27.1 \%)$ \\
\hline Billroth II & 35 (72.9\%) \\
\hline \multicolumn{2}{|l|}{ Previous cancer stage } \\
\hline Stage I & $30(62.5 \%)$ \\
\hline Stage II & $11(22.9 \%)$ \\
\hline Stage III & $7(14.6 \%)$ \\
\hline \multicolumn{2}{|l|}{ Previous PRM (cm) } \\
\hline$<3$ & $9(18.8 \%)$ \\
\hline$\geq 3$ & 39 (81.3\%) \\
\hline Total & $5.9 \pm 3.8(0.5-20)$ \\
\hline \multicolumn{2}{|l|}{ Previous LI } \\
\hline Positive & 17 (35.4\%) \\
\hline Negative & $31(64.6 \%)$ \\
\hline \multicolumn{2}{|l|}{ Previous VI } \\
\hline Positive & $3(6.3 \%)$ \\
\hline Negative & $45(93.8 \%)$ \\
\hline \multicolumn{2}{|l|}{ Recurrence site } \\
\hline Non-anastomosis & $21(43.8 \%)$ \\
\hline Anastomosis & $27(56.3 \%)$ \\
\hline \multicolumn{2}{|l|}{ Differentiation } \\
\hline Differentiated & $21(43.8 \%)$ \\
\hline Undifferentiated & $27(56.3 \%)$ \\
\hline \multicolumn{2}{|l|}{ Lauren classification } \\
\hline Intestinal & 17 (35.4\%) \\
\hline Diffuse & $18(37.5 \%)$ \\
\hline Mixed & $13(27.1 \%)$ \\
\hline \multicolumn{2}{|l|}{ RGC stage } \\
\hline Stage I & $24(50 \%)$ \\
\hline Stage II & $12(25 \%)$ \\
\hline Stage III & $12(25 \%)$ \\
\hline
\end{tabular}

$R G C$, remnant gastric cancer; $P R M$, proximal resection margin; $L I$, lymphatic invasion; $V I$, vascular invasion 
Table 2 Interval of RGC according to variables

\begin{tabular}{|c|c|c|c|c|}
\hline Variables & Interval (month) & Effect size (Cohen' d) & $95 \% \mathrm{Cl}$ & $p$ value \\
\hline \multicolumn{5}{|l|}{ Age (years) } \\
\hline$<65(n=25)$ & $92.4 \pm 67.7(14-256)$ & - & - & \multirow[t]{2}{*}{0.208} \\
\hline$\geq 65(n=23)$ & $119.8 \pm 80.7(14-350)$ & 0.369 & $-0.202,0.94$ & \\
\hline \multicolumn{5}{|l|}{ Sex } \\
\hline Male $(n=42)$ & $109.2 \pm 76.1(14-350)$ & - & - & \multirow[t]{2}{*}{0.372} \\
\hline Female $(n=6)$ & $79.8 \pm 63.7(15-179)$ & -0.393 & $-1.252,0.465$ & \\
\hline \multicolumn{5}{|l|}{ Previous cancer stage } \\
\hline Stage I $(n=30)$ & $103.0 \pm 80.1(14-350)$ & & - & \multirow[t]{3}{*}{0.810} \\
\hline Stage $\|(n=11)$ & $101.7 \pm 58.1(15-225)$ & -0.016 & $-0.707,0.673$ & \\
\hline Stage III $(n=7)$ & $122.8 \pm 81.6(28-256)$ & 0.247 & $-0.577,1.071$ & \\
\hline \multicolumn{5}{|l|}{ Previous PRM (cm) } \\
\hline$<3(n=9)$ & $66.0 \pm 77.3$ & - & - & \multirow[t]{2}{*}{0.077} \\
\hline$\geq 3(n=39)$ & $114.7 \pm 72.0$ & 0.667 & $-0.068,1.404$ & \\
\hline \multicolumn{4}{|l|}{ Previous LI } & \multirow[t]{3}{*}{0.857} \\
\hline Positive $(n=17)$ & $102.9 \pm 69.9$ & - & - & \\
\hline Negative $(n=31)$ & $107.1 \pm 78.3$ & 0.054 & $-0.536,0.646$ & \\
\hline \multicolumn{4}{|l|}{ Previous VI } & \multirow[t]{3}{*}{0.546} \\
\hline Positive $(n=3)$ & $80.0 \pm 60.25$ & - & - & \\
\hline Negative $(n=45)$ & $107.3 \pm 75.8$ & 0.362 & $-0.808,1.533$ & \\
\hline \multicolumn{5}{|l|}{ Reconstruction method } \\
\hline Billroth I $(n=13)$ & $67 \pm 34.5 .5(14-143)$ & - & - & \multirow[t]{2}{*}{0.003} \\
\hline Billroth II $(n=35)$ & $119.9 \pm 80.7(14-350)$ & 1.017 & $0.349,1.686$ & \\
\hline \multicolumn{5}{|l|}{ Recurrence site } \\
\hline Non-anastomosis $(n=21)$ & $70.8 \pm 53.6(14-256)$ & - & - & \multirow[t]{2}{*}{0.002} \\
\hline Anastomosis $(n=27)$ & $132.6 \pm 78.3(14-350)$ & 0.953 & $0.352,1.554$ & \\
\hline \multicolumn{5}{|l|}{ Differentiation } \\
\hline Differentiated $(n=21)$ & $125.6 \pm 87.1(14-350)$ & - & - & \multirow[t]{2}{*}{0.102} \\
\hline Undifferentiated $(n=27)$ & $90 \pm 60.6(14-221)$ & -0.485 & $-1.063,0.09$ & \\
\hline \multicolumn{5}{|l|}{ Lauren classification } \\
\hline Intestinal $(n=17)$ & $138.5 \pm 88.5(14-350)$ & - & - & \multirow[t]{3}{*}{0.044} \\
\hline Diffuse $(n=18)$ & $76.2 \pm 65.0(14-206)$ & -0.805 & $-1.495,-0.116$ & \\
\hline Mixed $(n=13)$ & $103.0 \pm 50.9(27-190)$ & -0.474 & $-1.207,0.257$ & \\
\hline \multicolumn{5}{|l|}{ RGC stage } \\
\hline Stage I $(n=24)$ & $109.7 \pm 84.2(14-350)$ & - & - & \multirow[t]{3}{*}{0.145} \\
\hline Stage $\|(n=12)$ & $72.0 \pm 47.3(15-190)$ & -0.605 & $-1.312,0.101$ & \\
\hline Stage III $(n=12)$ & $130.9 \pm 69.2(28-256)$ & 0.266 & $-0.429,0.961$ & \\
\hline
\end{tabular}

$R G C$, remnant gastric cancer; PRM, proximal resection margin; LI, lymphatic invasion; VI, vascular invasion; Calculation of Cohen's $d$, first row category is set to control group and lower row category is set to experimental group

II reconstruction (RGC-B II) $(p=0.003$, Cohen's $d=$ 1.017) (Table 2). Interval was categorized into lesser than 5 years group, more than 5 years but less than 10 years group, and more than 10 years group for more detailed comparison of interval according to reconstruction method. There were 5 (38.5\%) cases of RGC-B I and 10 (28.6\%) cases of RGC-B II in lesser than 5 years group, 7
(53.8\%) cases of RGC-B I and 8 (22.9\%) cases of RGC-B II in more than 5 years to less than 10 years group, and 1 (7.7\%) case of RGC-B I and 17 (48.6\%) cases of RGC-B II in more than 10 years group. Distribution of interval group of RGC-M according to each reconstruction method demonstrated statistically significant difference $(p=0.024, \mathrm{OR}=0.571,8.500)$. Also, there was 
significant difference in recurrence site according to previous reconstruction method $(p=0.001, \mathrm{OR}=13.750)$. Eleven (84.6\%) cases of RGC-B I recurred at nonanastomosis site and $2(15.4 \%)$ cases of RGC-B I recurred at anastomosis site. Ten (28.6\%) cases of RGC-B II recurred at non-anastomosis site and 25 (71.4\%) cases of RGC-B II recurred at anastomosis site. There was no significant difference in the advancement of previous gastric cancer $(p=0.151, \mathrm{OR}=5.789,3.272)$ or previous PRM $(p=0.580$, OR $=1.450)$ between RGC-B I and RGC-B II (Table 3).

\section{Discussion}

Although only a few studies have been performed on the incidence of RGC, RGC after gastric cancer (RGC-M) has increased due to increased survival rate of gastric cancer patients according to early detection and improvement in surgical technique and chemotherapy [8]. Many RGC studies have been conducted. However, there is no consensus on characteristics, range of resection, or prognosis of RGC due to problems such as the rareness of RGC and the inconsistency of definitions of RGC. In the current situation where RGC-M is expected to increase, this study is meaningful in that it evaluates characteristics of RGC-M and the occurrence pattern of RGC-M such as recurrence site and interval according to previous reconstruction method.

Most studies about the interval of RGC have reported that the interval of RGC-B is longer than that of RGC-M [9]. It has been proposed that this diversity of interval by initial disease originates from differences in mechanism of carcinogenesis. Environmental factors such as chronic

Table 3 Clinicopathologic features of RGC according to reconstruction method

\begin{tabular}{|c|c|c|c|c|c|c|}
\hline Variables & $\begin{array}{l}\text { RGC-BI } \\
(n=13)\end{array}$ & $\begin{array}{l}\text { RGC-B II } \\
(n=35)\end{array}$ & $p$ value $^{\dagger}$ & OR & $95 \%$ Cl for OR & $p$ value $^{\ddagger}$ \\
\hline \multicolumn{7}{|l|}{ Interval (years) } \\
\hline$<5$ & $5(38.5 \%)$ & $10(28.6 \%)$ & 0.024 & 1 & - & - \\
\hline$\geq 5,<10$ & $7(53.8 \%)$ & $8(22.9 \%)$ & & 0.571 & $0.130 \sim 2.503$ & 0.458 \\
\hline$\geq 10$ & $1(7.7 \%)$ & $17(48.6 \%)$ & & 8.500 & $0.865 \sim 83.493$ & 0.066 \\
\hline \multicolumn{7}{|l|}{ Recurrence site } \\
\hline Non-anastomosis & $11(84.6 \%)$ & $10(28.6 \%)$ & 0.001 & 1 & - & - \\
\hline Anastomosis & $2(15.4 \%)$ & $25(71.4 \%)$ & & 13.750 & $2.574 \sim 73.455$ & 0.002 \\
\hline \multicolumn{7}{|l|}{ Differentiation } \\
\hline Differentiated & $6(46.2 \%)$ & 15 (42.9\%) & 0.838 & 1 & - & - \\
\hline Undifferentiated & $7(53.8 \%)$ & $20(57.1 \%)$ & & 1.143 & $0.314 \sim 4.109$ & 0.838 \\
\hline \multicolumn{7}{|l|}{ Previous cancer stage } \\
\hline Stage I & $11(84.6 \%)$ & 19 (54.3\%) & 0.151 & 1 & - & - \\
\hline Stage II & $1(7.7 \%)$ & $10(28.6 \%)$ & & 5.789 & $0.651 \sim 51.505$ & 0.115 \\
\hline Stage III & $1(7.7 \%)$ & $6(17.1 \%)$ & & 3.272 & $0.369 \sim 32.743$ & 0.277 \\
\hline \multicolumn{7}{|l|}{ Previous PRM (cm) } \\
\hline$<3$ & $3(23.1 \%)$ & $6(17.1 \%)$ & 0.687 & 1 & - & - \\
\hline$\geq 3$ & $10(76.9 \%)$ & 29 (82.9\%) & & 1.450 & $0.304 \sim 6.909$ & 0.641 \\
\hline Total & $6.4 \pm 3.6$ & $5.7 \pm 3.9$ & 0.580 & & & \\
\hline \multicolumn{7}{|l|}{ Previous LI } \\
\hline Positive & $3(23.1 \%)$ & $14(40.0 \%)$ & 0.330 & 2.222 & $0.518 \sim 9.537$ & 0.283 \\
\hline Negative & $10(76.9 \%)$ & $21(60.0 \%)$ & & 1 & - & - \\
\hline \multicolumn{7}{|l|}{ Previous VI } \\
\hline Positive & $1(7.7 \%)$ & $2(5.7 \%)$ & 1.000 & 0.727 & $0.060 \sim 8.769$ & 0.802 \\
\hline Negative & $12(92.3 \%)$ & $33(94.3 \%)$ & & 1 & - & - \\
\hline \multicolumn{7}{|l|}{ Lauren classification } \\
\hline Intestinal & $4(30.8 \%)$ & $13(37.1 \%)$ & 0.904 & 1 & - & - \\
\hline Diffuse & $5(38.5 \%)$ & $13(37.1 \%)$ & & 0.800 & $0.174 \sim 3.669$ & 0.774 \\
\hline Mixed & $4(30.8 \%)$ & $9(25.7 \%)$ & & 0.692 & $0.136 \sim 3.518$ & 0.658 \\
\hline
\end{tabular}

$R G C$, remnant gastric cancer; $R G C-B$ I, remnant gastric cancer after Billroth I reconstruction; $R G C-B$ II, remnant gastric cancer after Billroth II reconstruction; $O R$, odds ratio; $P R M$, proximal resection margin; $L I$, lymphatic invasion; $V I$, vascular invasion; $p$ value ${ }^{t}, p$ value for chi-square test; $p$ value ${ }^{\ddagger}, p$ value for odds ratio 
stimulation by bile reflux and mucosa denervation after gastrectomy are responsible for RGC-B occurrence in remnant stomach $[10,11]$. These environmental changes can also affect remnant stomach after gastrectomy for malignant disease. In addition to environmental factors, remnant stomach after gastrectomy for malignant disease already has precancerous factors such as atrophic gastritis and intestinal metaplasia or hidden malignancy and metachronous gastric cancer in remnant stomach. A combination of these precancerous factors and environmental factors in remnant stomach after malignant disease contributes to the shorter interval of RGC-M [12]. That is, precancerous factors in remnant stomach are the main causes of diversity of interval between RGC-B and RGC-M.

The interval of RGC-B I is also significantly shorter than that of RGC-B II in most reported studies [13, 14]. In our study, although RGC-B was excluded, the mean interval of RGC-B I was 67 months (5.5 years), which was shorter than that (119 months or 9.9 years) of RGCB II $(p=0.003$, Cohen's $d=1.017)$ (Table 2). Both the interval of RGC-M and the recurrence site of RGC-M were related to previous reconstruction methods (Table $3)$. We found that RGC-B I showed frequent recurrences around the non-anastomotic site (11 out of 13, 84.6\%) while RGC-B II showed a tendency to often recur at the anastomotic site ( 25 out of $35,71.4 \%)(p=0.001$, OR = 13.750), consistent with results of other studies $[9,14]$ (Fig. 3). Considering the tendency of RGC to occur at different locations according to each reconstruction method, mechanisms of carcinogenesis in remnant stomach are different from those at anastomosis site after Billroth II and those at non-anastomosis site after Billroth I. Usually, the size of remnant stomach after Billroth I is larger than that after Billroth II to make for tension-free gastro-duodenostomy. Increase in the size of remnant stomach can raise the possibility of remnant stomach to have precancerous lesion with genetic vulnerability to RGC. Therefore, precancerous factor might be the main mechanism contributing to the carcinogenesis of RGC-B I with short interval at non-anastomosis site. In remnant stomach after Billroth II, it is considered that lower possibility of precancerous lesion and frequent bile reflux through gastrojejunostomy are mechanisms of carcinogenesis. Thus, the interval of RGC-B II at anastomosis site is longer than the interval of RGC-B I at non-anastomosis site due to weaker precancerous factor and stronger environmental factor than that after Billroth I reconstruction (Fig. 2).

As previously mentioned in the method, the final decision of the reconstruction method was done after visualization of the cancer lesion. Oncologic safety and tension-free anastomosis were decisive factors. To secure pathologically negative resection margin, 2 - to $3-\mathrm{cm}$ margin length is usually enough for early gastric cancer and over $5-\mathrm{cm}$ margin length is required for advanced gastric cancer. If the location of the cancer lesion is appropriate for tension-free gastro-duodenostomy with a) Remnant stomach after Billroth I reconstruction

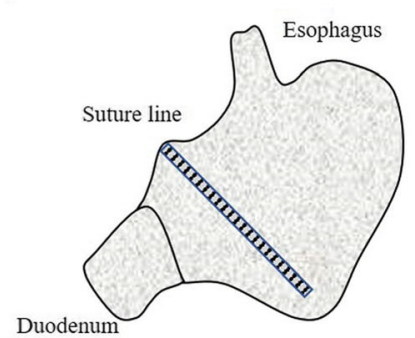

c) Remnant stomach after Billroth II reconstruction

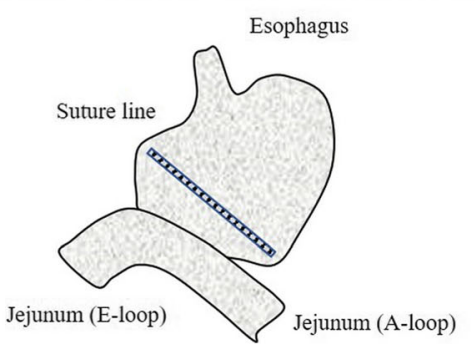

b) Distribution of RGC-B I in remnant stomach $(\mathrm{n}=13$, mean interval $=67$ months $)$

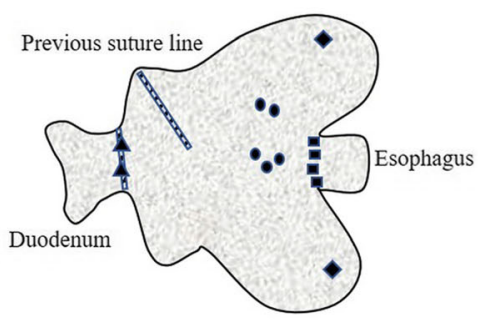

d) Distribution of RGC-B II in remnant stomach $(\mathrm{n}=35$, mean interval $=119.9$ months $)$

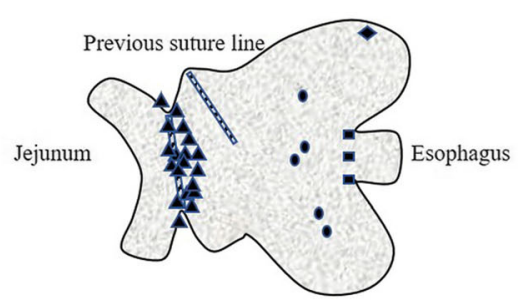

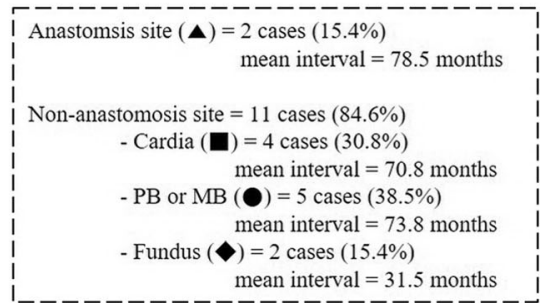

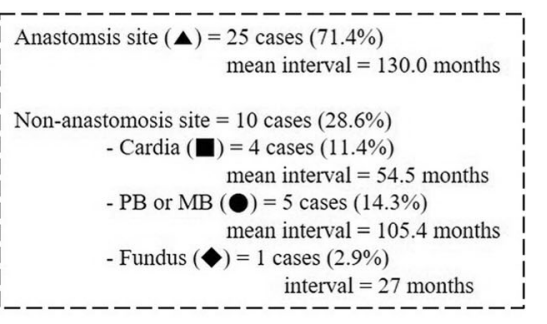

Fig. 2 Schematic figure of remnant stomach and the pattern of RGC according to previous reconstruction methods. Anastomosis sites are apart from suture line (transected line) (a), (c). Most (84.6\%) of RGC-B I occurred at non-anastomosis site, especially cardia and PB or MB (b). Most (71.4\%) of RGC-B II occurred at anastomosis site with long interval (130 months) (d). RGC, remnant gastric cancer; PB, proximal body; MB, midbody; RGC-B I, RGC after Billroth I reconstruction; RGC-B II, RGC after Billroth II reconstruction 
enough safety margin, Billroth I is preferred as a reconstruction method. If not, then Billroth II is preferred as a reconstruction method. Although previous reconstruction method was different, there was no significant difference in the length of previous PRM between RGC-B I and RGC-B II $(p=0.580)$ (Table 3$)$. If the resection margin is pathologically negative, the length of the resection margin does not affect the prognosis and local recurrence of gastric cancer patients $[15,16]$. In this study, there was no significant difference in the interval of RGC by the length of previous PRM ( $p=0.077$, Cohen's $d=0.667$ ) (Table 2). Thus, it is thought that the interval of RGC occurrence is not influenced by the length of pathologically negative PRM.

Surprisingly, there was no correlation between stages of the previous gastric cancer and the interval of RGCM ( $p=0.810$, Cohen's $d=-0.016,0.247)$ in this study (Table 2). In other words, the invasion depth of the previous gastric cancer or the degree of $\mathrm{LN}$ metastasis did not significantly affect the interval of RGC-M. Thus, we presume that the interval of RGC in remnant stomach is not influenced by the stage of previous gastric cancer, but by mechanisms of carcinogenesis such as environmental factors and precancerous factors. However, more data and genetic mutational studies on carcinogenesis of RGC-M are needed to prove this hypothesis. Also, more active surveillance will be needed after gastrectomy in patients with family history of gastric cancer or genetic mutation.

Many studies have been conducted on clinicopathologic features of RGC, reporting that RGC-M has several distinguishing features compared with primary gastric cancer. In RGC-M patients, there could be changes in lymphatic flow around remnant stomach by previous LN dissection. Such lymphatic flow changes might lead to alteration of LN metastasis pattern [17]. Also, the number of retrieved LN during RGC surgery is smaller than the number of retrieved LN during D2 dissection for primary gastric cancer. Thus, N category of TNM system for primary gastric cancer can not reflect the prognosis or the degree of LN metastasis in RGC-M patients. Although several studies have reported modified staging system using LN ratio, the utility of these alternative systems is not superior to TNM system for primary gastric cancer $[18,19]$. Considering these disease distinctions of RGC, another staging system and surgical guideline for LN dissection or extent of gastric resection are needed. Guideline of surgery and staging system for RGC are essential for correct analysis and comparison of data. Further well-designed large-scale studies on genetic mutation of RGC patients are possible on the basis of these foundations.

Open total gastrectomy was performed in this study. However, laparoscopic approach has been performed as a surgical option for RGC in other studies. Laparoscopic total gastrectomy for RGC has lesser blood loss and fewer post-op complications than the open approach. The 5-year survival rate of RGC patients with laparoscopic total gastrectomy was similar to that of patients with open total gastrectomy [20, 21]. However, there are only a few studies on the laparoscopic surgery of RGC. In addition, their sample sizes were small. Thus, further studies with large sample size are required.

Some researchers have found that the prognosis of early RGC is not worse than that of early primary gastric cancer, although the prognosis of advanced RGC is worse than that of advanced primary gastric cancer [22]. To obtain good prognosis of RGC, it is important not only to create new staging system and surgical guideline for RGC, but also to perform regular surveillance after gastrectomy for early diagnosis and treatment of RGC.

In the author's institution, surveillance after gastrectomy has been carried out annually until the 7th year for early gastric cancer and the 10th year for advanced gastric cancer. Thereafter, surveillance study was done every two years. Considering the facts that more than one-third of RGC occurred more than 10 years after the initial gastrectomy and there was no correlation between the interval of RGC-M and stage of previous gastric cancer, regular surveillance should be performed annually after the 10th year of gastrectomy for early diagnosis and treatment of RGC. Considering the distribution and interval of RGC in remnant stomach based on previous reconstruction methods (Fig. 3), we should observe carefully cardia and lesser curvature from early period of surveillance in patients with Billroth I reconstruction. Additional focus on anastomosis site is needed from the late period of surveillance in both patients with Billroth I reconstruction and patients with Billroth II reconstruction.

This study has several limitations. First, Roux-en-Y reconstruction, a commonly used reconstruction method, was not included in this study. In most studies comparing Roux-en-Y with Billroth reconstruction methods, remnant stomach after Roux-en-Y reconstruction showed lower incidence of reflux gastritis than Billroth reconstruction methods [23, 24]. Thus, it is thought that environmental factor after Roux-en-Y reconstruction is weaker than that after Billroth reconstruction. In addition, the size of remnant stomach after Roux-en-Y reconstruction is smaller than that after Billroth I reconstruction. Hence, precancerous factor after Roux-en-Y reconstruction seems to be weaker than that after Billroth I reconstruction. These different mechanisms of Roux-en-Y reconstruction with discrepancies in environmental and precancerous factors might be helpful in comprehending mechanisms of carcinogenesis in remnant stomach. Second, this study was a retrospective 


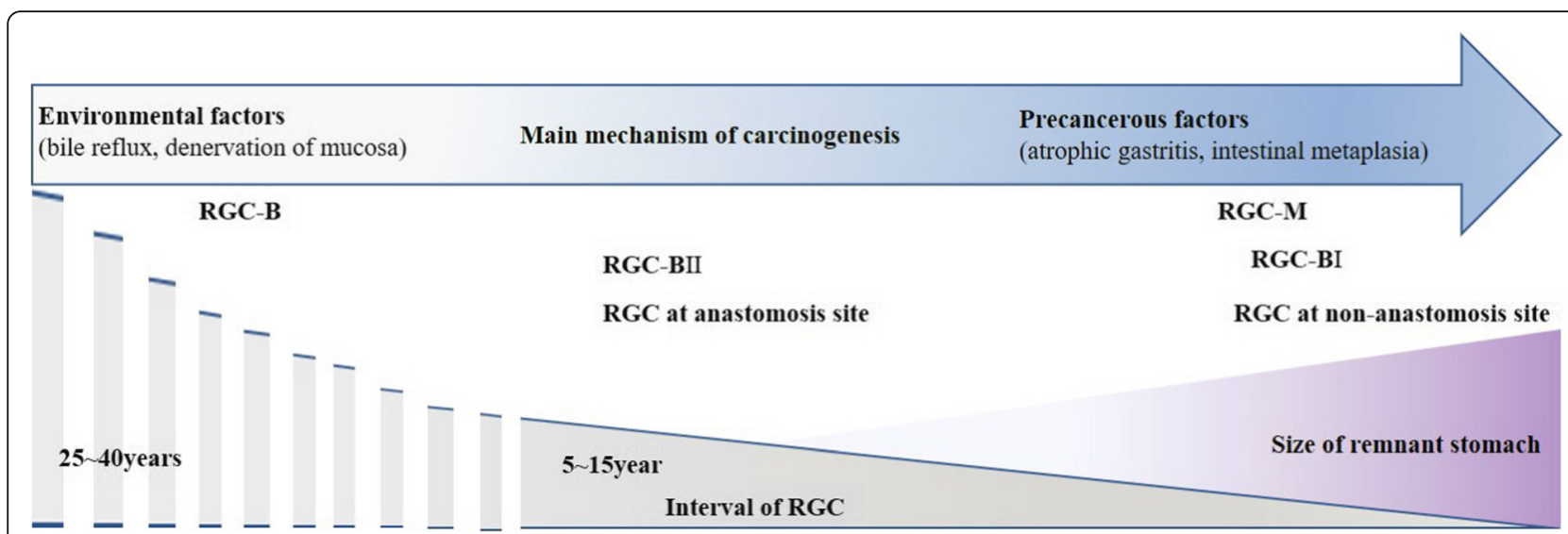

Fig. 3 Interval distribution of RGC according to diverse factors. Occurrence pattern and interval of RGC are affected by precancerous and environmental factors. These mechanisms of carcinogenesis are also affected by primary disease, previous reconstruction method, and size of remnant stomach. RGC, remnant gastric cancer; RGC-B, RGC after benign disease; RGC-M, RGC after gastric cancer; RGC-B I, RGC after Billroth I reconstruction; RGC-B II, RGC after Billroth II reconstruction

small sample sized study with selection bias. Unfortunately, we could not match clinicopathologic features affecting carcinogenesis in both reconstruction method groups due to small sample size. Hence, this study has limitation in the reliability of results. Based on results of this study, we have started a large-scale multi-center study with five tertiary referral university hospitals in Daegu, Korea (Daegu Gastric Cancer Study Group) from December 2018. Third, detailed analysis of splenectomy and metastasis pattern of $\mathrm{LN}$, especially mesenteric LN and LN 10, was not performed in this study. Thus, it is difficult to conclude whether there are beneficial effects of survival following splenectomy, dissection of mesenteric LN, or LN 10 stations. Fourth, we found that the interval and recurrence site of RGC were affected by multifarious factors involving precancerous and environmental factors. However, we could not perform genetic studies to prove mechanisms of carcinogenesis in remnant stomach in this study. Therefore, further largescale, well-designed study with genetic examinations is needed to understand the precise mechanism of carcinogenesis in remnant stomach.

\section{Conclusion}

RGC after Billroth I reconstruction tends to arise earlier at non-anastomosis site than RGC after Billroth II. Therefore, we should examine non-anastomosis site carefully from the beginning of surveillance after gastric cancer surgery with Billroth I reconstruction for better outcome.

\section{Abbreviations}

RGC: Remnant gastric cancer; YUMC: Yeungnam University Medical Center; RGC-B: Remnant gastric cancer after benign disease; RGC-M: Remnant gastric cancer after gastric cancer; EGD: Esophagogastroduodenoscopy; OR: Odds ratio; PRM: Proximal resection margin; RGC-B I: Remnant gastric cancer after
Billroth I reconstruction; RGC-B II: Remnant gastric cancer after Billroth II reconstruction; PB: Proximal body; MB: Mid-body

Acknowledgements

Not applicable

\section{Author' contributions}

YEP and SWK planned and designed the study. YEP collected the data. YEP and SWK analyzed and interpreted the patient data. SWK commented on drafts of the paper. YEP was a major contributor in writing the manuscript. All authors read and approved the final manuscript.

\section{Funding}

The authors declare that they have no funding.

\section{Availability of data and materials}

The anonymized data used and/or analyzed during the current study are available from the corresponding author on reasonable request.

\section{Ethics approval and consent to participate}

This study was approved by the Institutional Review Board of Yeungnam University Medical Center, Daegu, Republic of Korea (IRB No. 2018-09-030001).

\section{Consent for publication}

Not applicable

\section{Competing interests}

The authors declare that they have no competing interests.

Received: 4 August 2019 Accepted: 4 November 2019

Published online: 30 November 2019

\section{References}

1. Bray F, Ferlay J, Soerjomataram I, Siegel RL, Torre LA, Jemal A. Global cancer statistics 2018: GLOBOCAN estimates of incidence and mortality worldwide for 36 cancers in 185 countries. CA Cancer J Clin. 2018;68(6):394-424.

2. Jung KW, Won YJ, Kong HJ, Oh CM, Cho H, Lee DH, et al. Cancer statistics in Korea: incidence, mortality, survival, and prevalence in 2012. Cancer Res Treat. 2015:47(2):127-41.

3. Balfour DC. Factors influencing the life expectance of patients operated on for gastric ulcer. Ann Surg. 1922;76:405-8.

4. Sinning C, Schaefer N, Standop J, Hirner A, Wolff M. Gastric stump carcinoma - epidemiology and current concepts in pathogenesis and treatment. Eur J Surg Oncol. 2007;33(2):133-9. 
5. Lu J, Huang CM, Zheng CH, Li P, Xie JW, Wang JB, et al. Prognostic value of tumor size in patients with remnant gastric cancer: is the seventh UICC stage sufficient for predicting prognosis? PLoS One. 2014;9(12):e115776.

6. Ahn HS, Kim JW, Yoo MW, Park DJ, Lee HJ, Lee KU, et al. Clinicopathological features and surgical outcomes of patients with remnant gastric cancer after a distal gastrectomy. Ann Surg Oncol. 2008;15(6):1632-9.

7. Ohashi M, Katai H, Fukagawa T, Gotoda T, Sano T, Sasako M. Cancer of the gastric stump following distal gastrectomy for cancer. Br J Surg. 2007;94(1): 92-5.

8. Takeno S, Noguchi T, Kimura Y, Fujiwara S, Kubo N, Kawahara K. Early and late gastric cancer arising in the remnant stomach after distal gastrectomy. Eur J Surg Oncol. 2006;32(10):1191-4.

9. Takeno S, Hashimoto T, Maki K, Shibata R, Shiwaku H, Yamana I, et al. Gastric cancer arising from the remnant stomach after distal gastrectomy: a review. World J Gastroenterol. 2014:20(38):13734-40.

10. Kondo K. Duodenogastric reflux and gastric stump carcinoma. Gastric Cancer. 2002;5(1):16-22.

11. Kaminishi M, Shimizu N, Shimoyama S, Yamaguchi H, Tsuji E, Aoki F, et al. Denervation promotes the development of cancer-related lesions in the gastric remnant. J Clin Gastroenterol. 1997;25(Suppl 1):S129-34.

12. Ohira M, Toyokawa T, Sakurai K, Kubo N, Tanaka H, Muguruma K, et al. Current status in remnant gastric cancer after distal gastrectomy. World J Gastroenterol. 2016;22(8):2424-33.

13. Komatsu S, Ichikawa D, Okamoto K, Ikoma D, Tsujiura M, Nishimura Y, et al. Progression of remnant gastric cancer is associated with duration of followup following distal gastrectomy. World J Gastroenterol. 2012;18(22):2832-6.

14. Zhang DW, Dong B, Li Z, Dai DQ. Clinicopathologic features of remnant gastric cancer over time following distal gastrectomy. World I Gastroenterol. 2015;21(19):5972-8.

15. Kim MG, Lee JH, Ha TK, Kwon SJ. The distance of proximal resection margin dose not significantly influence on the prognosis of gastric cancer patients after curative resection. Ann Surg Treat Res. 2014;87(5):223-31.

16. Lee CM, Jee YS, Lee JH, Son SY, Ahn SH, Park DJ, et al. Length of negative resection margin does not affect local recurrence and survival in the patients with gastric cancer. World J Gastroenterol. 2014;20(30):10518-24.

17. Li F, Zhang R, Liang H, Liu H, Quan J, Zhao J. The pattern of lymph node metastasis and the suitability of 7th UICC N stage in predicting prognosis of remnant gastric cancer. J Cancer Res Clin Oncol. 2012;138(1):111-7.

18. Nakagawa M, Choi YY, An JY, Hong JH, Kim JW, Kim HI, et al. Staging for remnant gastric cancer: the metastatic lymph node ratio vs. the UICC 7th edition system. Ann Surg Oncol. 2016;23(13):4322-31.

19. Son SY, Kong SH, Ahn HS, Park YS, Ahn SH, Suh YS, et al. The value of $N$ staging with the positive lymph node ratio, and splenectomy, for remnant gastric cancer: a multicenter retrospective study. J Surg Oncol. 2017;116(7): 884-93

20. Kwon IG, Cho I, Guner A, Choi YY, Shin HB, Kim HI, et al. Minimally invasive surgery for remnant gastric cancer: a comparison with open surgery. Surg Endosc. 2014;28(8):2452-8.

21. Booka E, Kaihara M, Mihara K, Nishiya S, Handa K, Ito Y, et al. Laparoscopic total gastrectomy for remnant gastric cancer: a single-institution experience. Asian J Endosc Surg. 2019;12(1):58-63.

22. Shimada H, Fukagawa T, Haga Y, Oba K. Does remnant gastric cancer really differ from primary gastric cancer? A systematic review of the literature by the Task Force of Japanese Gastric Cancer Association. Gastric Cancer. 2016; 19(2):339-49.

23. Xiong JJ, Altaf K, Javed MA, Nunes QM, Huang W, Mai G, et al. Roux-en-Y versus Billroth I reconstruction after distal gastrectomy for gastric cancer: a meta-analysis. World J Gastroenterol. 2013;19(7):1124-34.

24. Shim JH, Oh SI, Yoo HM, Jeon HM, Park CH, Song KY. Roux-en-Y gastrojejunostomy after totally laparoscopic distal gastrectomy: comparison with Billorth II reconstruction. Surg Laparosc Endosc Percutan Tech. 2014; 24(5):448-51.

\section{Publisher's Note}

Springer Nature remains neutral with regard to jurisdictional claims in published maps and institutional affiliations.

\section{Ready to submit your research? Choose BMC and benefit from:}

- fast, convenient online submission

- thorough peer review by experienced researchers in your field

- rapid publication on acceptance

- support for research data, including large and complex data types

- gold Open Access which fosters wider collaboration and increased citations

- maximum visibility for your research: over $100 \mathrm{M}$ website views per year

At BMC, research is always in progress.

Learn more biomedcentral.com/submissions 